\title{
Performance Analysis of RIP, OSPF, IGRP and EIGRP Routing Protocols in a Network
}

\author{
Pankaj Rakheja \\ Assistant Professor \\ ITM University, School of \\ Engineering \& Tech, \\ Dept. of EECE, Gurgaon \\ (Haryana), India
}

\author{
Prabhjot kaur \\ Assistant Professor \\ ITM University, School of \\ Engineering \& Tech, \\ Dept. of EECE, Gurgaon \\ (Haryana), India
}

\author{
Anjali gupta \\ Third year B.Tech \\ students \\ ITM University, School of \\ Engineering \& Tech, \\ Dept. of EECE, Gurgaon \\ (Haryana), India
}

\author{
Aditi Sharma \\ Third year B.Tech \\ students \\ ITM University, \\ School of \\ Engineering \& \\ Tech, Dept. of \\ EECE, Gurgaon \\ (Haryana), India
}

\begin{abstract}
In a network topology for forwarding packets various routing protocols are being used. Routers maintain a routing table for successful delivery of the packets from the source node to the correct destined node. The extent of information stored by a router about the network depends on the algorithm it follows. Most of the popular routing algorithms used are RIP, OSPF, IGRP and EIGRP. Here in this paper we are analyzing the performance of these very algorithms on the basis of the cost of delivery, amount of overhead on each router, number of updates needed, failure recovery, delay encountered and resultant throughput of the system. We are trying to find out which protocol suits the best for the network and through a thorough analysis we have tried to find the pros and cons of each protocol.
\end{abstract}

\section{INTRODUCTION}

Internet protocol suite is used for communication for the internet and similar networks. TCP/IP [1-4] provides end to end connectivity and also specifies how data should be formatted, addressed, transmitted, routed and received at the destination. It basically has four abstraction layers namely link layer, internet layer, transport layer and application layers. Each layer has its own functions as Link Layer contains communication technologies for a local network; Internet Layer connects local networks and establishes internetwork communication; Transport layer handles host to host communication and Application layer contains protocols specific for data communication services on process to process level.

Data is encapsulated that is Enclosing of a set of data (code) into another set of code to protect its integrity when transferring it between non-compatible systems, or to secure it from unauthorized access during transmission the figure below shows data encapsulation; it shows how the data is encapsulated from application layer to link layer via transport and internet layers. At each layer a set of header and footer are added and at the receiver side it is unwrapped that is header and footer are removed right from link layer first to application layer to get the data or message transmitted by the sender.

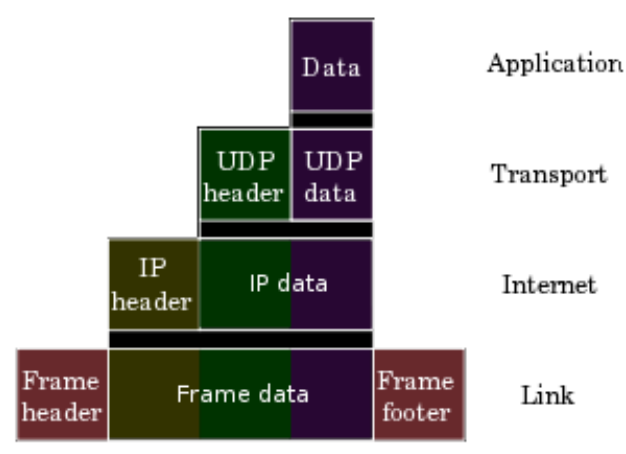

Fig 1:Data encapsulation in TCP/IP suit

The figure 2 below shows the data movement from the source to the destination via different layers and through intermediate nodes which may are generally routers.

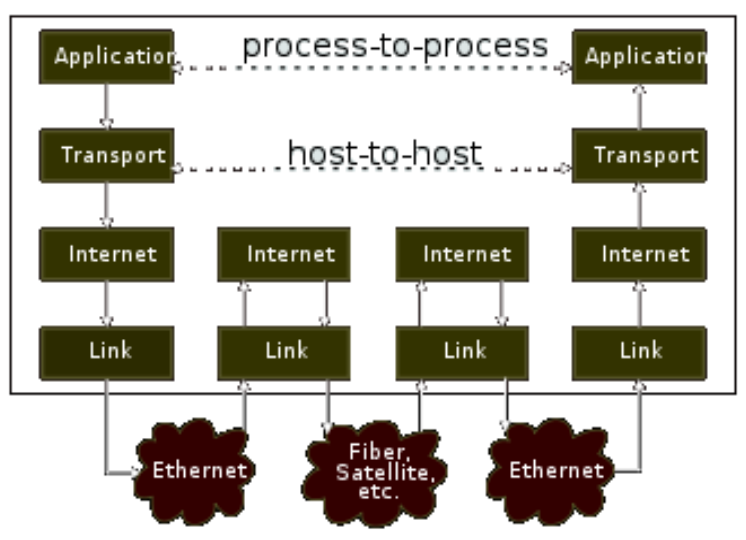

Fig 2:Data Flow from source to destination

The figure 3 below shows a simple link between sender $\mathrm{A}$ and receiver $\mathrm{B}$ via routers. 


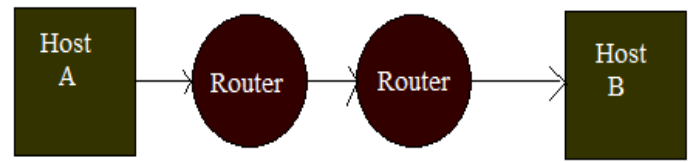

Fig 3:Two hosts connected via routers in TCP/IP

Here in this paper we are concentrating on internetworking which is the function of the internet layer. This is enabled by router which is a device that forwards data packets between computer networks, creating an overlay internetwork. It is connected to two or more data lines from different networks. When a data packet comes in on one of the lines, the router reads the address information in the packet to determine its ultimate destination. Then, using information in its routing table or routing policy, it directs the packet to the next network on its journey. It is a data table stored in a router or a networked computer that lists the routes to particular network destinations, and in some cases, metrics (distances) associated with those routes. They contain information about the topology of the network immediately around it. The construction of routing tables is the primary goal of routing protocols. This paper comprises of five sections. The first one comprises of introduction of $\mathrm{TCP} / \mathrm{IP}$ protocol and communication in a network, second one comprises of brief overview of various protocols being compared, in the third section includes the designed scenarios, fourth section consists of the results obtained and last section comprises of the conclusion drawn from the results obtained.

\section{OVERVIEW}

\subsection{Open Shortest Path First}

OSPF [5] [7] [9] is a routing protocol which was defined as version 2 in RFC 2328; used to allow routers to dynamically learn routes from other routes and to advertise routes to other routes; advertisements containing routes are referred to as link state advertisements; keeps the track of all the various links between itself and a $\mathrm{n} / \mathrm{w}$ it is trying to send data to; summarizes the route information, reduces the number advertised routes and reduces the n/w load, uses a designated router to reduce the quantity and frequency of link state advertisements; it has a router, processor, memory more than other routing protocols; selects the best routes of finding the lowest cost paths to a destination; Protocol suite: TCP/IP; Protocol type: Transport layer interior link state routing protocol; Interior Gateway Protocol: Routing Information in an autonomous system; Link State based Algorithm: The state of the interface or link is used to decide the path on which the information is routed; Multiple links with same state is possible. Demand to a destination can be routed on multiple paths.

\subsubsection{ROUTING USING OSPF}

Routers maintain database with link state information, weights computed using link state, IP address etc. This database in each router is updated by transmitting Link State Advertisements throughout the autonomous system. A shortest path tree is constructed by each router with itself as the root node and based on weights in the database.

\subsection{ROUTING INFORMATION PROTOCOL}

The Routing Information Protocol (RIP) [5][8][10][11] is a distance-vector protocol that uses hop count as its metric. The Routing Information Protocol (RIP) provides the standard IGP protocol for local area networks, and provides great network stability, guaranteeing that if one network connection goes down the network can quickly adapt to send packets through another connection. It is widely used for routing traffic in the global Internet and is an interior gateway protocol (IGP), which means that it performs routing within a single autonomous system. RIP itself evolved as an Internet routing protocol, and other protocol suites use modified versions of RIP. IP RIP is formally defined in two documents: Request For Comments (RFC) 1058 and 1723. RFC 1058 (1988) describes the first implementation of RIP, while RFC 1723 (1994) updates RFC 1058. RFC 1058 enables RIP messages to carry more information and security features.

\subsubsection{RIP UPDATES}

RIP sends routing-update messages at regular intervals and when the network topology changes. When a router receives a routing update that includes changes to an entry, it updates its routing table to reflect the new route. The metric value for the path is increased by one, and the sender is indicated as the next hop. RIP routers maintain only the best route (the route with the lowest metric value) to a destination. After updating its routing table, the router immediately begins transmitting routing updates to inform other network routers of the change. These updates are sent independently of the regularly scheduled updates that RIP routers send. The algorithm that enables each router to update its database with the fastest route communicated from neighboring routers:

Database each RIP router on a given network keeps a database that stores the following information for every computer in that network:

- IP Address. The Internet Protocol address of the computer.

- Gateway. The best gateway to send a message addressed to that IP address.

- Distance: The number of routers between this router and the router that can send the message directly to that IP address.

- Route change flag. A flag that indicates that this information has changed, used by other routers to update their own databases.

- Timers. Various timers.

The RIP algorithm works like this

- Update. At regular intervals each router sends an update message describing its routing database to all the other routers that it is directly connected to. Some routers will send this message as often as every 30 seconds, so that the network will always have up-todate information to quickly adapt to changes as computers and routers come on and off the network.

- Propagation. When a router $X$ finds that a router $Y$ has a shorter and faster path to a router $\mathrm{Z}$, then it will update its own routing database to indicate that fact. Any faster path is quickly propagated to neighboring 
routers through the update process, until it is spread across the entire RIP network.

Each hop in a path from source to destination is assigned a hop-count value, which is typically 1 . When a router receives a routing update that contains a new or changed destinationnetwork entry, the router adds one to the metric value indicated in the update and enters the network in the routing table. The IP address of the sender is used as the next hop.

\subsection{INTERIOR GATEWAY ROUTING PROTOCOL}

The Interior Gateway Routing Protocol (IGRP)[5-8] [10-11] is a routing protocol to provide routing within an autonomous system (AS). Distance-vector routing protocols calls for each router to send all or a portion of its routing table in a routingupdate message at regular intervals to each of its neighboring routers. As routing information proliferates through the network, routers can calculate distances to all nodes within the internetwork. IGRP adheres to the following Distance-Vector characteristics: sends out periodic routing updates (every 90 seconds); sends out the full routing table every periodic update; uses a form of distance as its metric; uses the Bellman-Ford Distance Vector algorithm to determine the best "path" to a particular destination; supports only IP routing; utilizes IP protocol 9; routes have an administrative distance of 100; by default, supports a maximum of 100 hops. This value can be adjusted to a maximum of 255 hops; is a classful routing protocol; uses Bandwidth and Delay of the Line, by default, to calculate its distance metric. Reliability, Load, and MTU are optional attributes that can be used to calculate the distance metric; requires that you include an Autonomous System (AS) number in its configuration. Only routers in the same Autonomous system will send updates between each other.

\subsection{Enhanced Interior Routing Protocol}

Enhanced Interior Gateway Routing Protocol (EIGRP) or Enhanced IGRP [5-11] is a Cisco proprietary routing protocol utilizing the Diffusing Update Algorithm (DUAL). EIGRP is a hybrid protocol as it incorporates features of a Distance Vector routing protocol and features of a Link State routing protocol. Enhanced Interior Gateway Routing Protocol (EIGRP) is an enhanced version of IGRP. used in TCP/IP and OSI internets. It is regarded as an interior gateway protocol (IGP) but has also been used extensively as an exterior gateway protocol for inter-domain routing. Key capabilities that distinguish Enhanced IGRP (EIGRP) from other routing protocols include fast convergence, support for variablelength subnet mask, support for partial updates, and support for multiple network layer protocols. A router running EIGRP stores all its neighbors' routing tables so that it can quickly adapt to alternate routes. If no appropriate route exists, EIGRP queries its neighbors to discover an alternate route. These queries propagate until an alternate route is found.

The support of EIGRP for variable-length subnet masks permits routes to be automatically summarized on a network number boundary. In addition, EIGRP can be configured to summarize on any bit boundary at any interface.

\subsubsection{WORKING}

\subsubsection{Neighbor Table}

Each router keeps state information about adjacent neighbors. When newly discovered neighbors are learned, the address and interface of the neighbor is recorded. This information is stored in the neighbor data structure. The neighbor table holds these entries. There is one neighbor table for each protocol dependent module. When a neighbor sends a hello, it advertises a HoldTime. The HoldTime is the amount of time a router treats a neighbor as reachable and operational. In other words, if a hello packet isn't heard within the HoldTime, then the HoldTime expires. When the HoldTime expires, DUAL is informed of the topology change.

The neighbor table entry also includes information required by the reliable transport mechanism. Sequence numbers are employed to match acknowledgments with data packets. The last sequence number received from the neighbor is recorded so out of order packets can be detected. A transmission list is used to queue packets for possible retransmission on a per neighbor basis. Round trip timers are kept in the neighbor data structure to estimate an optimal retransmission interval.

\subsubsection{Topology Table}

The Topology Table is populated by the protocol dependent modules and acted upon by the DUAL finite state machine. It contains all destinations advertised by neighboring routers. Associated with each entry is the destination address and a list of neighbors that have advertised the destination. For each neighbor, the advertised metric is recorded. This is the metric that the neighbor stores in its routing table. If the neighbor is advertising this destination, it must be using the route to forward packets. This is an important rule that distance vector protocols must follow. Also associated with the destination is the metric that the router uses to reach the destination. This is the sum of the best advertised metric from all neighbors plus the link cost to the best neighbor. This is the metric that the router uses in the routing table and to advertise to other routers.

\subsubsection{Feasible Successors}

A destination entry is moved from the topology table to the routing table when there is a feasible successor. All minimum cost paths to the destination form a set. From this set, the neighbors that have an advertised metric less than the current routing table metric are considered feasible successors. Feasible successors are viewed by a router as neighbors that are downstream with respect to the destination. These neighbors and the associated metrics are placed in the forwarding table. When a neighbor changes the metric it has been advertising or a topology change occurs in the network, the set of feasible successors may have to be re-evaluated. However, this is not categorized as a route recomputation. When a link to a neighbor that is the only feasible successor goes down, all routes through that neighbor commence a route recomputation and enter the Active state 


\section{SCENARIO DESIGNED}

We have designed a network comprising of slip8_gateway connected via PPP_DS3 links. The figure 4 shows the designed scenario where the terrain size is $15 \mathrm{sq} \mathrm{km}$, traffic is created between A-F, I-D, H-K, E-G and B-J. We have analyzed the performance of various protocols in terms of cost of packet delivery and overhead over routers. The figure 5 shows the scenario where a router fails then we observed and analyzed the delivery of packets to destined node. The figure 6 shows the cost of each link is obtained using the formula that $\mathrm{Cost}=($ Reference bandwidth/ link bandwidth $)$.

The various protocols we analyzed are RIP, OSPF, IGRP and EIGRP respectively. Then for OSPF we have divided the network into areas. OSPF-area 1 is confined to communicate within a given area whereas in OSPF- area inter network communication is allowed.

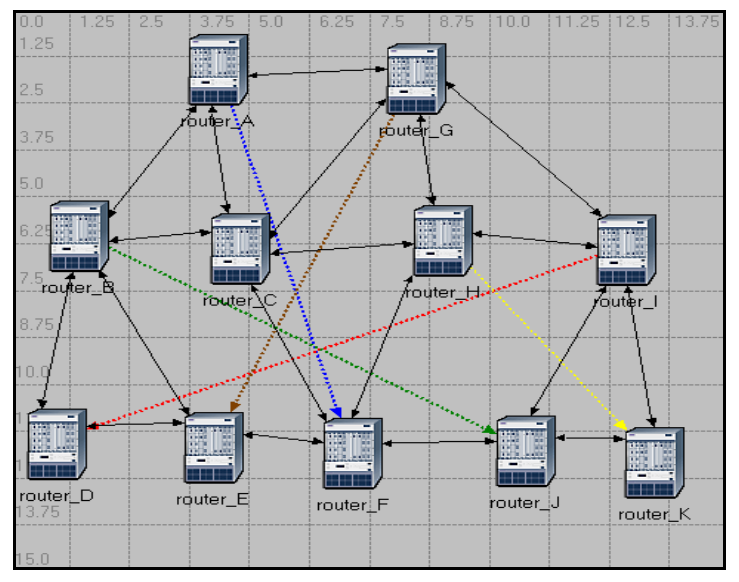

Fig 4:Screenshot of the network designed

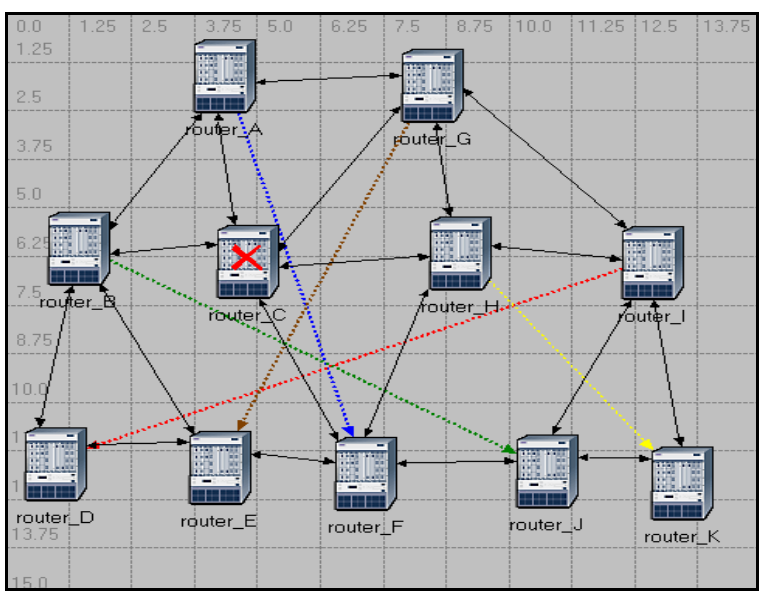

Fig 5:Screenshot of the scenario with failed router

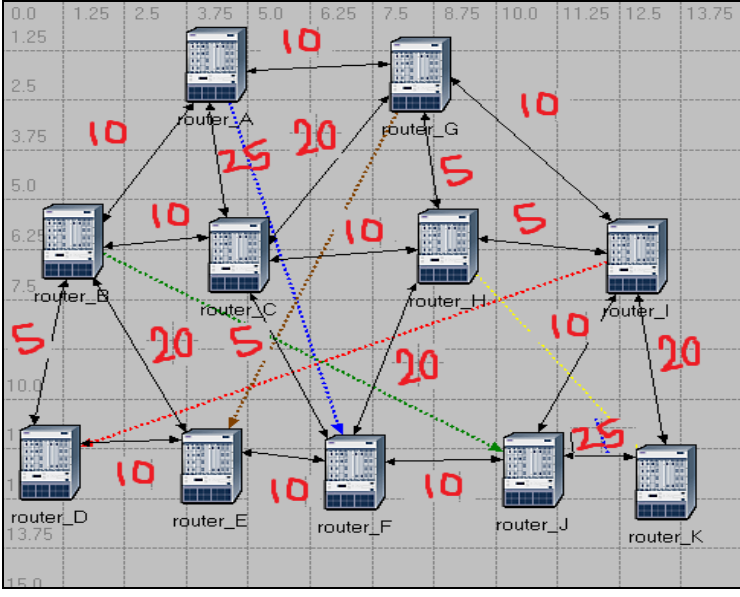

Fig 6:Screenshot of scenario showing cost of each link

\section{RESULTS}

We have analyzed the performance of various routing protocols naming RIP, OSPF, IGRP and EIGRP over a scenario of size $15 \mathrm{sq} \mathrm{km}$ consisting of slip8_gateway routers and on simulating the network we obtained the following results for best effort traffic which are shown below in table 1 which shows cost of transmission between two routers for different protocols. We also have analyzed overhead on routers and overall performance in terms of throughput, queuing delay and link utilization figures 7-12 show the results obtained.

TABLE I. COMPARISON ON BASIS OF COST OF DELIVERY

\begin{tabular}{|l|l|l|l|l|l|l|}
\hline Protocol & OSPF & $\begin{array}{l}\text { OSPF- } \\
\text { area1 }\end{array}$ & $\begin{array}{l}\text { OSPF- } \\
\text { area2 }\end{array}$ & RIP & IGRP & EIGRP \\
\hline Application & & & & & & \\
\hline A-F & 25 & nil & 25 & 30 & 40 & 25 \\
\hline I-D & 30 & nil & 40 & 45 & 30 & 40 \\
\hline H-K & 25 & 25 & 25 & 25 & 25 & 25 \\
\hline E-G & 30 & nil & 30 & 35 & 40 & 35 \\
\hline B-J & 25 & nil & 25 & 40 & 25 & 25 \\
\hline
\end{tabular}

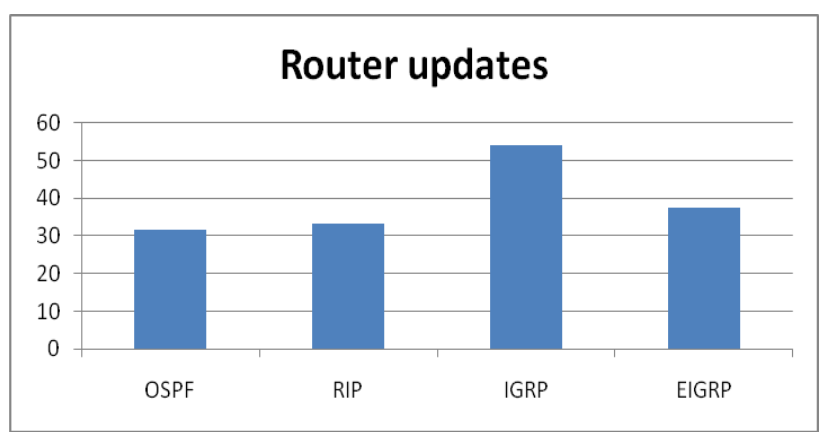

Fig 7:Router updates comparision of various protocols 


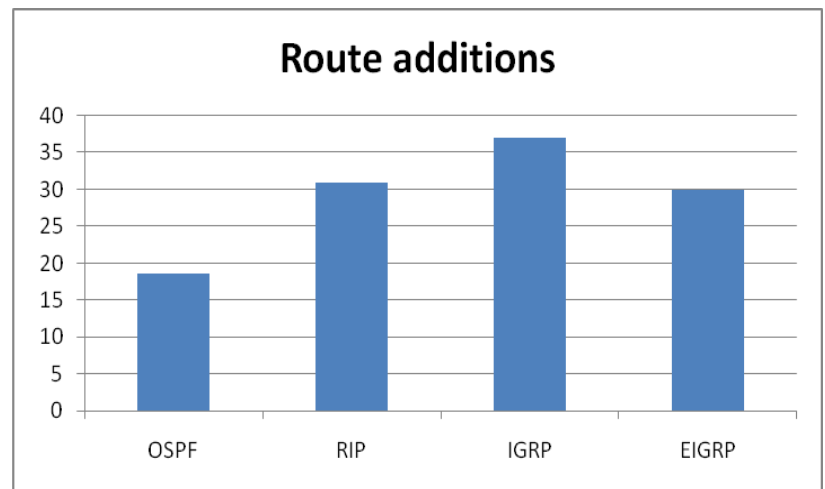

Fig 8:Route additions comparision of various protocols

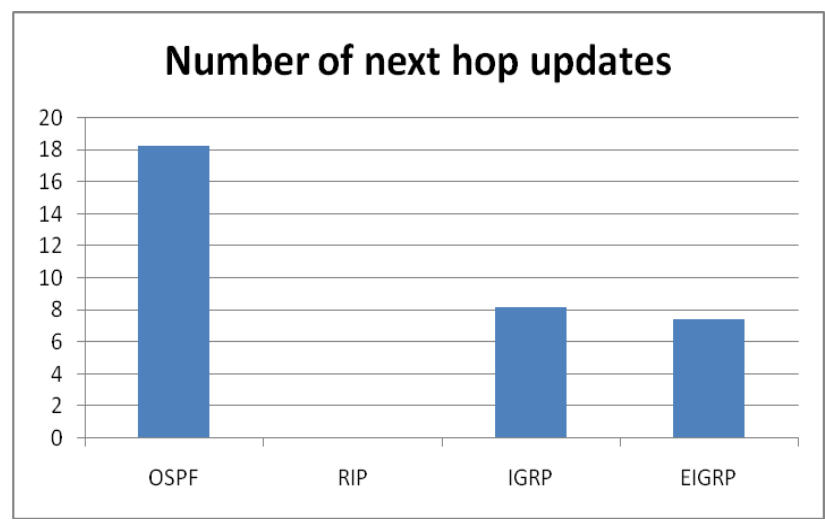

Fig 9:Number of next hop updates of various protocols

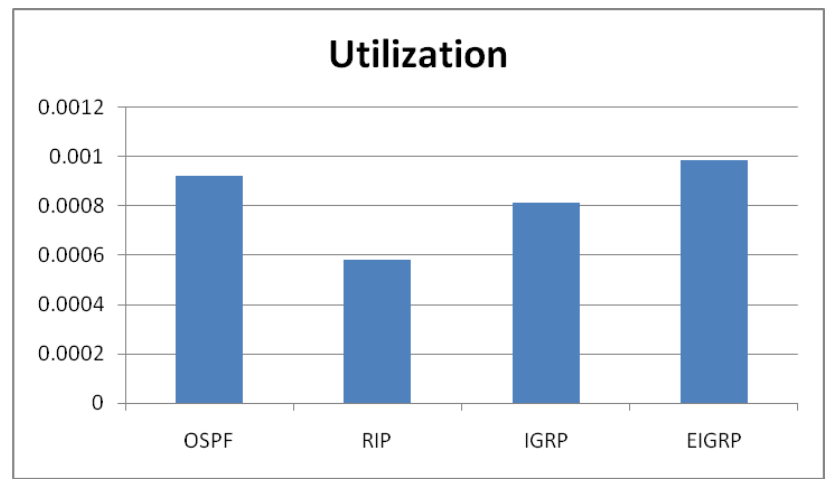

Fig 10: Link utilization of various protocols

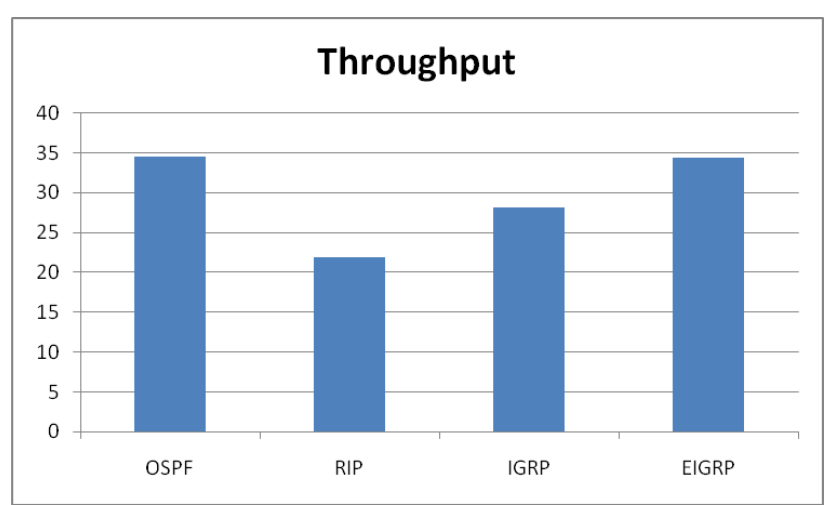

Fig 11:Throughput of various protocols

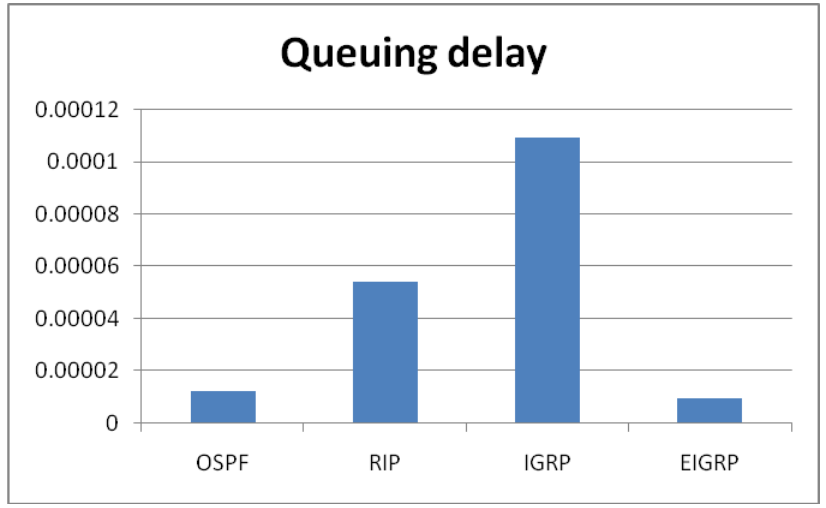

Fig 12:Delay encoutered by various protocols

As per table 1 OSPF has the least cost of transmission followed by EIGRP, IGRP and RIP. In case of router overhead shown in figure 7-9 IGRP has the maximum overhead followed by EIGRP, OSPF and RIP. And on analyzing the performance parameters like throughput, utilization and delay, as per results plotted OSPF has the maximum throughput followed by EIGRP, IGRP and RIP shown in figure 10; for the case of queuing delay EIGRP has the least delay followed by OSPF,RIP and IGRP shown in figure 12 and for the case of link utilization EIGRP has the maximum link utilization followed by OSPF, IGRP and RIP as shown in figure 11 .

\section{CONCLUSION}

On analyzing the result of the performance of various routing protocols naming RIP, OSPF, IGRP and EIGRP over a scenario for cost of transmission, router overhead, throughput, link utilization and queuing delay we can say that OSPF has best performance overall as it has the least cost of transmission, lower router overhead after RIP and maximum throughput amongst all routing protocol and queuing delay of it is second lowest after EIGRP and it also has second highest link utilization after EIGRP. Then EIGRP performs good as it has cost of transmission just above OSPF and has optimum router over head and overall performance in terms of throughput, Queuing delay and link utilization.

So for best effort service that is transmission of data packets OSPF performs better than other protocols for throughput, queuing delay, utilization and overhead.

\section{REFERENCES}

[1] Youngmi Joo,Vinay Ribeirot,Anja Feldmannt,Anna C.Gilbert,and Walter Willinger Guang Yang,", "TCP/IP traffic dynamics and network performance: A lesson in workload modeling, flow control,and trace-driven simulations",ACMSIGCOM

[2] Guang Yang, "Introduction to TCP/IP Network Attacks" white paper available seclab.cs.sunysb.edu/sekar/papers/netattacks.pdf

[3] Rajesh. R, Lakshmanan. M and Noor Mohammed “: Implementation of Networked Control Systems using TCP/IP” , International Journal of Computer Applications 18(2):1-5, March 2011.

[4] M. Z. Rashed, Ahmed E. Hassan \& Ahmed I. Sharaf, "MODEL BASED SYSTEM ENGINEERING APPROACH OF A LIGHTWEIGHT EMBEDDED 
TCP/IP", International Journal of Computer Science \& Information Technology (IJCSIT), Vol 3, No 2, April 2011

[5] Wu, Bing, "Simulation Based Performance Analyses on RIP, EIGRP and OSPF Using OPNET"

[6] Vishal sharma, Rajneesh Narula and Sameer khullar "Performance Analysis of IEEE 802.3 using IGRP and EIGRP Routing Protocols" International Journal of Computer Applications (0975 - 8887)Volume 44- No13, April 2012

[7] Ittiphon krinpayorm and Suwat Pattaramalai,"Link Recovery Comparison Between OSPF \& EIGRP ", International Conference on Information and Computer Networks (ICICN 2012) IPCSIT vol. 27 (2012) IACSIT Press, Singapore
[8] Mr. R. M. Pethe, Miss S. R .Burnase TECHNICAL ERA LANGUAGE OF THE NETWORKING - EIGRP International Journal of Engineering Science and Technology (IJEST) NCICT Special Issue Feb 2011

[9] Mehboob Nazim Shehzad, Najam-Ul-Sahar, "Simulation of OSPF Routing Protocol Using OPNET Module"(A Routing Protocol Based on the Link-State Algorithm)

[10] Bernard Fortz,Jennifer Rexford and Mikkel Thorup. Traffic Engineering With Traditional IP Routing Protocols." IEEE Communications Magazine. October 2002, pp. 118-124.

[11] Ahmad Karim, Minhaj Ahmad Khan "Behaviour of Routing Protocols for Medium to Large Scale Networks", Australian Journal of Basic and Applied Sciences, 5(6): 1605-1613, 2011 